\title{
Can mental rotation begin before perception finishes?
}

\author{
ERIC RUTHRUFF \\ University of Califormia, San Diego, La Jolla, California \\ and \\ JEFF MILLER \\ University of Otago, Dunedin, New Zealand
}

\begin{abstract}
We conducted six experiments to determine if mental rotation can begin before perception finishes, as allowed by continuous flow models but not discrete stage models of information processing. The results of Experiments 1-3 showed that the effect of shape discriminability on RT was underadditive with the effect of stimulus orientation, suggesting that mental rotation began before shape discrimination had finished and that the two processes overlapped in time. The results of Experiments 4-6 indicated that mental rotation can overlap with color discriminations as well. In both sets of experiments, however, the amount of underadditivity tended to be much less than predicted by models allowing interference-free overlap. This suggests that mental rotation can overlap with perceptual analysis, contrary to fully discrete models, but that little rotation is carried out during this overlap due to interference between simultaneous discrimination and rotation processes.
\end{abstract}

There is general agreement that mental processing is composed of functionally distinct stages (e.g., Cohen \& Squire, 1980; Damasio, 1985; McClelland, 1979; Sanders, 1980; Sternberg, 1969). However, many fundamental questions regarding the architecture of human cognition remain to be answered. For example, how many stages of processing are there in a given task? Are they performed in a strict sequence or with some temporal overlap?

Sternberg (1969) proposed that stages operate discretely, such that each stage begins once the previous stage has finished. This model, shown in Figure 1a, makes specific predictions regarding the types of factors that will show additive effects in factorial experiments, as formalized in the "additive factor method" of Sternberg (1969). Experiments employing this method have yielded a consistent set of results, which, arguably, can be taken as support for the underlying discrete model (Sanders, 1980, 1983; Sternberg 1975).

Despite the successes of the additive factor method, discrete models have come under attack from many di-

During the course of this research, the first author received support from a Nation Science Foundation Graduate Fellowship and Public Health Service Training Grant 2T32-MH14268. This research was also supported by National Institute of Mental Health Grant PHSMH40733 to J.M. We would like to thank Soledad Ballesteros, Tom Carr, Aaron Ilan, Craig Kunimoto, Geoff Loftus, Toby Mordkoff, Hal Pashler, and Tom Sanocki for helpful comments on an earlier version of the manuscript. We also thank Shakha Vora and Nina Constantino for their help in running the experiments. Correspondence concerning this article should be addressed to E. Ruthruff, Department of Psychology, University of California-San Diego, La Jolla, CA 92093-0109, or to J. Miller, Department of Psychology, University of Otago, P.O. Box 56, Dunedin, New Zealand. rections. Some have claimed that discrete processing is implausible given that the brain is composed of highly interconnected systems of neurons, rather than the sort of encapsulation that is perhaps suggested by discrete models. Critics have also claimed that such models are incompatible with evidence of graded effects of certain experimental variables (e.g., Eriksen \& Schultz, 1979; Norman \& Bobrow, 1975) and with evidence of overlapping stages (e.g., Shaffer, 1971). Although some theorists argue that these criticisms are unfounded or grossly overstated (Meyer, Osman, Irwin, \& Yantis, 1988; Miller, 1988; Sanders, 1990), discrete models are now often regarded as being overly simplistic (e.g., Rabbitt, 1986).

An alternative model is shown in Figure 1b. This model belongs to the class of models we will refer to as continuous flow models (e.g., the cascade model of McClelland, 1979), because information is transmitted continuously from one stage to the next (see Eriksen \& Schultz, 1979). Because information is transmitted continuously, a stage can begin operating even before its predecessor has finished, and several stages may thus be active simultaneously. This property, which we refer to as temporal overlap between stages, is one of the most important characteristics distinguishing continuous models from discrete models (see Miller, 1988, for a discussion of other differences between these models).

Many of the published attempts to discriminate between discrete stage and continuous flow models have asked whether the perceptual process transmits information continuously to the response preparation processthat is, whether there is temporal overlap between perception and response preparation (e.g., Coles, 1989; Miller, 
a)

Discrete

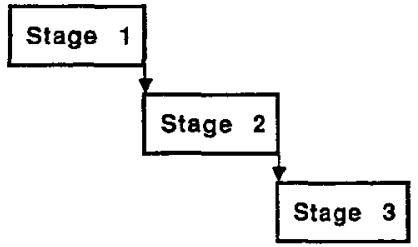

b)

Continuous

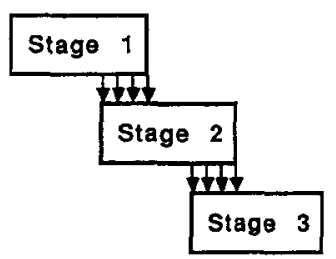

TIme

Figure 1. Discrete stage and continuous flow models of information processing.

1982, 1983, 1987; Miller \& Hackley, 1992; Osman, Bashore, Coles, Donchin, \& Meyer, 1992; Smid, Lamain, Hogeboom, Mulder, \& Mulder, 1991). In some cases, response preparation does appear to begin before perception has finished (as predicted by continuous flow models), but, in other cases, it does not (as predicted by discrete models). After reviewing many of these studies, Miller (1988) concluded that the results could not be explained by strictly discrete or strictly continuous models. He favored the alternative asynchronous discrete coding (ADC) model (Miller, 1982), which asserts that only separately coded pieces of information (e.g., identity and color) can be independently transmitted from one stage to the next. Thus, it is possible for stage $N$ to be working on one or more of the distinct codes, while stage $N-1$ is still working on the other code(s).

Although the experiments described above indicate that perception and response preparation do overlap to some degree, we cannot yet make generalizations regarding other cognitive processes. In particular, it is not safe to assume that the flow of information is equally discrete or continuous between all pairs of stages. For instance, information might flow continuously from perceptual to decision processes but discretely from decision to response processes. Clearly, it will be necessary to study information flow between different sets of processes in order to find out whether one particular form of information transmission characterizes all or most of the information processing sequence and, if so, what its properties are.

The primary goal of the present experiments was to determine whether perceptual discrimination processes (specifically, shape and color discrimination) must finish before the onset of mental rotation, a process thought to involve the transformation of a mental image from one orientation to another. We chose to study mental rotation for several reasons. Most importantly, mental rotation is a relatively clear-cut example of a distinct mental process, and its basic operation is understood well enough that we can expect to manipulate it effectively (Shepard \& Cooper, 1982). Furthermore, mental rotation is a slow, effortful process, so its duration can be varied over a range of several hundred milliseconds. This allows us to more easily test the predictions of models allowing temporal overlap between stages.

In the experiments to be described below, subjects viewed stimulus letters and determined if the letters were displayed in their normal or mirror image forms. Previous research with this task suggests that subjects mentally rotate an image of the stimulus letters into the upright orientation before making the mirror/normal judgment (Cooper \& Shepard, 1973); thus, by varying stimulus orientation, we can vary the duration of mental rotation processes. Subjects also discriminated between different shapes (Experiments 1-3) or colors (Experiments 4-6), and the difficulty of these discriminations varied between blocks. ${ }^{1}$

In principle, mental rotation can begin once the subject has formed a rotatable image (and knows in which direction to rotate) and, under certain circumstances, it should be possible for this image to be formed before completion of the required shape or color discriminations. Thus, it is logically possible for mental rotation to overlap with perceptual discrimination. However, overlap will occur only if the perceptual process transmits information nondiscretely to the mental rotation processthat is, if the perceptual process transmits preliminary information about stimulus shape (i.e., the rotatable image) to the mental rotation process while it is still working on the required perceptual discriminations. If instead the perceptual process transmits information discretely, after it has finished forming the rotatable image and making the additional perceptual discriminations, then temporal overlap between mental rotation and perception should not be possible.

In Experiments 1-3, we attempted to determine if mental rotation can overlap with shape discriminations; in Experiments 4-6, we tried to determine if mental rotation can overlap with color discriminations. Mental rotation and form discrimination both operate upon stimulus shape, whereas mental rotation and color discrimination operate upon distinct stimulus attributes. Therefore, a comparison between the two sets of experiments allows us to determine if the amount of temporal overlap is influenced by the similarity of the types of information being manipulated or by the similarity of the operations needed for mental rotation and the required discrimination.

\section{Predictions of Temporal Overlap Models}

In order to determine whether mental rotation overlaps with perception, the present experiments employed a diagnostic based on the additivity or underadditivity of 
factor effects in a factorial reaction time (RT) experiment (see Egeth \& Dagenbach, 1991; Schweickert \& Townsend, 1989; Stanovich \& Pachella, 1977). One factor was the orientation of the stimulus (in degrees from vertical), which affects the duration of mental rotation processes. The second factor was the difficulty of the required perceptual discrimination. In Experiment 1, for example, an easy discrimination required subjects to discriminate $y$ from $r$, whereas a more difficult discrimination required them to distinguish between the letters $y$ and $g$, which were visually similar in the stimulus font used.

A wide variety of RT models predict that two factors will have additive effects if they influence processes that are carried out in strict sequence, yet they have underadditive effects if they influence processes that are carried out at the same time (see Miller, 1993; Schweickert \& Townsend, 1989; Townsend \& Ashby, 1983). Thus, if mental rotation can begin only after shape discrimination has been completed-as assumed by discrete models--then orientation and the difficulty of the letter discrimination should have additive effects on RT. Conversely, if mental rotation can begin before letter identification has been completed and can proceed in parallel with it-as allowed by continuous models-then orientation and discriminability should interact underadditively.

The prediction of additivity for the discrete stage model is straightforward and has been described elsewhere (e.g., Sternberg, 1969); the prediction of underadditivity for temporally overlapping processes, meanwhile, is somewhat more complicated. To illustrate the prediction, we will first suppose that processing times are deterministic. Processing time diagrams for an overlap model with deterministic processing times are shown in Figure 2 for upright and upside-down stimuli. A hypothetical plot of mean RTs resulting from such a model is shown at the bottom of Figure 2. When the stimulus is upright, very little, if any, mental rotation will be required, as shown at the top of Figure 2, and prolonging perception should thus have a large effect. These conditions produce the two points on the left side of the graph. But when the stimulus is upside-down and mental rotation takes a long time, as shown in the middle of Figure 2, prolonging perception should have no effect on the latency of the overt response. These conditions produce the two identical points on the right side of the graph. Thus, this model predicts that the discriminability effect will disappear completely for upside-down stimuli, resulting in a large underadditive interaction between orientation and discriminability.

The predictions of the overlap model are much the same when we allow for variability in processing times, although the discriminability effect cannot be expected to disappear entirely for upside-down stimuli in this case. As discussed next, the discriminability effect at each orientation depends upon the exact characteristics of the overlap model (e.g., whether simultaneous perceptual and mental rotation processes interfere with each other), but, for virtually all such models, the dis-
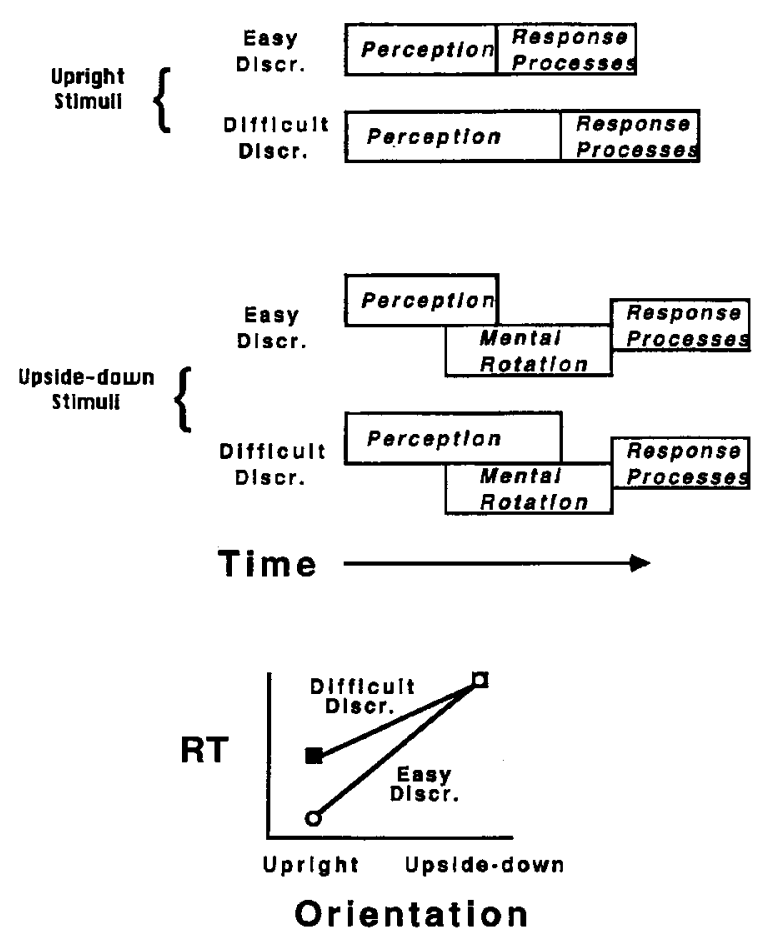

Figure 2. Processing time diagrams for upright and upside-down stimuli, with temporal overlap between mental motation and perception, and a hypothetical plot of reaction time as a function of discrimination difficulty and orientation.

criminability effect should be smaller for upside-down stimuli than for upright stimuli.

The most extreme continuous flow models predict what we will call interference-free overlap. In these models, perception and mental rotation not only operate simultaneously but each operates at the same rate as it would if it were performed in isolation (i.e., there is no interference between simultaneous processes). To compute the underadditivity predicted by interference-free overlap models, it is necessary to assume a specific joint probability distribution of the times needed for perception and mental rotation. Unfortunately, even the marginal distributions of these times are unobservable, and therefore, any specific assumption is necessarily somewhat arbitrary. To overcome this problem, we tried a large range of plausible assumptions, computing the underadditivity predicted in each case. Although this basic approach is simple, the procedures behind these computations are complicated. Therefore, we present only a sketch of the procedure here; a complete discussion of the computations can be found in the Appendix.

In brief, we first inferred limits on the parameters of the marginal distributions from observed RTs. Then, we computed the predicted underadditivities throughout the space of possible combinations of parameter values. For example, if we inferred that the mental rotation rate $\left(P_{1}\right)$ was between 300 and $500 \mathrm{msec}$ per $180^{\circ}$ and the mean discrimination time $\left(P_{2}\right)$ was between 150 and $250 \mathrm{msec}$, we would examine predictions for 121 different joint 
distributions with all possible combinations of $P_{1}$ and $P_{2}$ from the sets $\{300,310,320 \ldots 500\}$ and $\{150,160$, $170 \ldots 250\}$, respectively, under various assumptions about the correlation present in the joint distribution. Using this process, we determined what range of interaction sizes can be predicted by plausible interferencefree overlap models. The minimum and maximum of these predicted interactions are shown in Table 1, for each experiment.

Less extreme continuous flow models, which will be called partial overlap models, allow mental rotation to overlap with discrimination, but they assume that only a small amount of rotation can be accomplished during the overlap. There are several reasons why one might expect partial, rather than interference-free, overlap. First, the perceptual system might not transmit the necessary outputs to mental rotation at the exact moment they become available. If these outputs are delayed, the amount of overlap will be less than the maximum possible. Furthermore, even if the proper codes are immediately transmitted to the mental rotation stage, interference might arise when mental rotation and perception must operate concurrently (e.g., due to capacity limitations, outcome conflicts). Such interference could slow either or both processes relative to their rates in isolation and thereby reduce the amount of underadditivity. For example, mental rotation might engage a single-channel mechanism that is also required to carry out a subset of the addition shape or color discriminations, in which case mental rotation could overlap at most partially with perception.

Because partial overlap models are not uniquely specified, it is difficult to determine how much underadditivity they predict in general. However, it seems likely that plausible partial overlap models would predict less underadditivity than would interference-free overlap models. Therefore, when we found evidence indicating that there was some temporal overlap of perceptual discrimination and mental rotation in the present experiments, we then tried to determine whether the amount of underadditivity was large enough to be consistent with interference-free overlap models, or whether it was instead small enough to suggest that some type of inter-

Table 1

Predicted and Observed Deviations From Additivity for Experiments 1-6

\begin{tabular}{|c|c|c|c|c|}
\hline \multirow[b]{3}{*}{ Experiment } & \multirow{2}{*}{\multicolumn{2}{|c|}{ Predicted }} & \multicolumn{2}{|r|}{ Observed } \\
\hline & & & & $95 \%$ Confidence \\
\hline & Minimum & Maximum & $M$ & Interval \\
\hline 1 & 69 & 126 & 51 & $15-87$ \\
\hline 2 & 113 & 162 & 52 & $13-91$ \\
\hline 3 & 54 & 128 & -30 & $-64-4$ \\
\hline 4 & 21 & 25 & 33 & $6-60$ \\
\hline 5 & 91 & 104 & 41 & $-9-51$ \\
\hline 6 & 62 & 74 & 24 & $-15-63$ \\
\hline
\end{tabular}

Note-Positive numbers indicate underadditivity; negative numbers indicate overadditivity. The predictions were derived from models allowing interference-free temporal overlap between perception and mental rotation (see Appendix). ference had occurred, consistent with partial overlap models. Specifically, we determined if the observed underadditivity was significantly smaller than the minimum predicted by the interference-free overlap models (i.e., the data were too nearly additive). If so, we rejected these models and concluded that some interference must have been present.

In summary, by examining the effects of discriminability and orientation on RT in a factorial design, we sought to determine if mental rotation and perception are performed in strict sequence, with interference-free temporal overlap or with partial overlap. Discrete models predict additive effects, whereas models allowing interference-free overlap predict large underadditive interactions. The predictions of partial overlap models, meanwhile, lie somewhere between those of the two extreme models. In Experiments $1-3$, we examined the possibility of overlap between mental rotation and shape discrimination; in Experiments 4-6, we looked for overlap between mental rotation and color discrimination.

\section{EXPERIMENT 1}

Experiment 1 was an initial attempt to determine if mental rotation can begin before shape perception has finished, using the additivity/underadditivity diagnostic described above. Subjects viewed single stimulus letters (mirror-image or normal versions) presented in different orientations across trials. Stimulus discriminability was varied by pairing within a block either two visually similar letters (e.g., $y$ and $g$ ) or two visually dissimilar letters (e.g., $y$ and $r$ ). The subjects' task was to rapidly determine both the identity and the version (mirror-image vs. normal) of the letter, pressing one of two keys to indicate the response. The stimulus-response (S-R) mapping was chosen so that subjects had to know both the identity and version of the letter before preparing a response. In a block with $y$ s and gs, for example, subjects pressed one key if they saw a normal $y$ or a mirror-image $g$, and they pressed the other key if they saw a normal $g$ or a mirror-image $y$.

\section{Method}

Subjects. Sixty-four undergraduates at the University of California, San Diego, participated in Experiment 1 in partial fulfillment of a course requirement. Five subjects were replaced because they failed to meet our criterion of $80 \%$ correct. $^{2}$

Stimuli. The stimuli were produced by IBM-PC-compatible computers connected to NEC Multisync display monitors, viewed from a distance of about $60 \mathrm{~cm}$. The stimuli were the lowercase letters $y, g, r$, and $t$ and their mirror images, drawn in light blue on a white background. The letter fonts were chosen so that the letter pairs $y, g$ and $r, t$ were visually similar. Letters were presented in one of six possible orientations: $0^{\circ}, 60^{\circ}, 120^{\circ}, 180^{\circ}, 240^{\circ}$, or $300^{\circ}$ clockwise from upright.

Procedure. Prior to each block of trials, the subjects were told which two letters they would see during that block. They were instructed to identify each stimulus letter and determine whether it was a normal or mirror-image version. Responses were made by pressing the " $z$ " key or the " $/$ " key. The "/" key was assigned to the normal version of one letter and the mirror-image version of the 
other letter, and the " $z$ " key was assigned to the complementary versions of each of the two letters. The assignment of stimuli to response keys was counterbalanced across subjects. The subjects were encouraged to respond rapidly while making very few mistakes.

The discriminability of the letter pairs varied between blocks. In the difficult discrimination blocks, the subjects discriminated between visually similar letters. The subjects participated in two difficult discrimination blocks: a $y, g$ block and an $r, t$ block, the order of which was counterbalanced across subjects. In the two easy discrimination blocks, the subjects distinguished between visually dissimilar letters (e.g., $y$ and $r$ ). The letters were selected by first choosing either $y$ or $g$ and then choosing either $r$ or $t$; thus, there were four possible pairs of letters $(y, r ; y, t ; g, r ; g, t)$. Two of these pairs were chosen for each subject such that each letter was used exactly once per subject in the easy discrimination blocks and such that each of the four possible letter pairs was used equally often across subjects. Half of the subjects participated in the difficult block first, and half participated in the easy block first. The subjects then alternated between easy and difficult blocks until they had completed all four blocks. Each subject saw each letter in two different blocks: once in an easy block and once in a difficult block. To avoid confusion, the normal and mirror-image versions of the letter were mapped to the same responses in both blocks.

On each trial, stimulus identity, version, and orientation were chosen randomly, with the restriction that each combination of orientation, version, and stimulus identity occur equally of ten within a block of trials. The trial began with the presentation of a fixation cross for $600 \mathrm{msec}$. Three hundred milliseconds after the cross was removed, the stimulus appeared at fixation and remained present until a response was made. The computer then indicated whether the response was correct or incorrect, and the next trial began $1 \mathrm{sec}$ later.

The experimental session consisted of four blocks of 144 trials and lasted about $45 \mathrm{~min}$. The subjects were given 20 randomly selected practice trials before the first block and 8 randomly selected practice trials before each subsequent block.

\section{Results}

Mean RTs and error rates are shown in Figure 3 as a function of stimulus orientation and discriminability. Throughout the experiments reported in this article, we found equivalent effects of clockwise and counterclockwise rotations and have therefore combined the data from complementary orientations (e.g., $90^{\circ}$ and $270^{\circ}$ ).

The large effect of orientation on RT was highly reliable $[F(3,186)=276.60, p<.001],{ }^{3}$ as was its effect on error rates $[F(3,186)=36.80, p<.001]$. The much smaller effect of discriminability $(28 \pm 35 \mathrm{msec}),{ }^{4}$ was not statistically reliable. Figure 4 shows the mean discriminability effect on RT, and the $95 \%$ confidence interval around it, as a function of stimulus orientation (the data from all six experiments are presented together in this graph to facilitate comparison). The discriminability effect decreases monotonically with increasing orientation, indicating an underadditive interaction between orientation and discriminability. This interaction effect (measured as the difference in discriminability effects to upright and upside-down stimuli) was $51 \pm$ $36 \mathrm{msec}$, which is statistically reliable. Furthermore, a linear trend analysis ${ }^{5}$ on RTs, which is arguably a sensitive test for the form of underadditivity predicted by models allowing temporal overlap, indicated a significantly steeper increase in RT across orientations in the
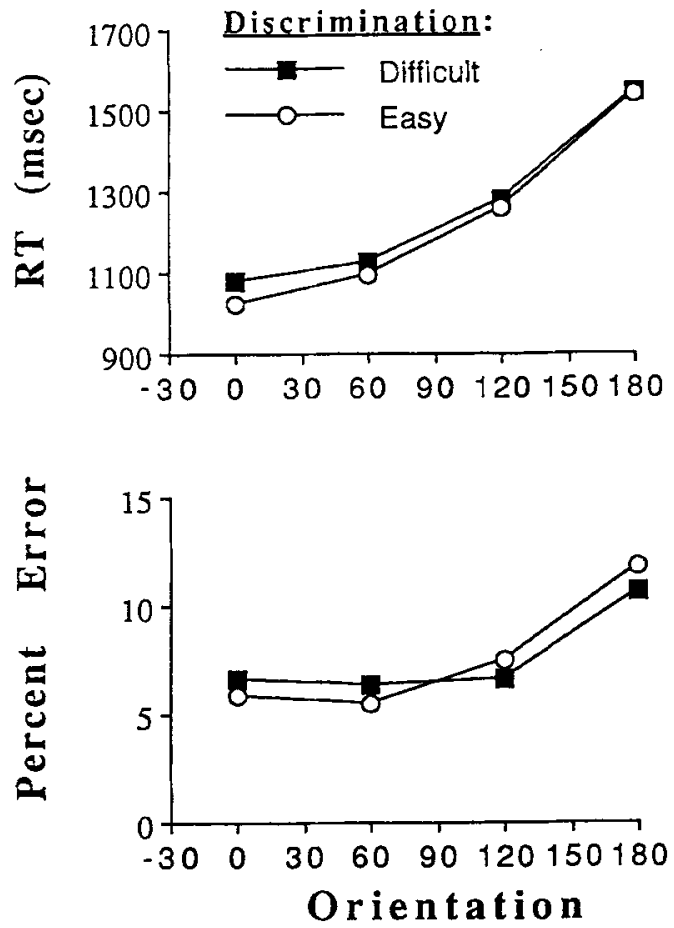

Figure 3. Mean reaction time and percent error for Experiment 1 as a function of discrimination difficulty and orientation.

easy discrimination condition $[F(1,62)=4.01, p<.05]$. The significant underadditivity suggests that the perceptual discrimination and mental rotation overlapped in time, contrary to discrete models.

As can be seen in Figure 3, the discriminability effect nearly disappears for upside-down stimuli, which seems consistent with models allowing interference-free overlap between perception and mental rotation. This was confirmed by computing predictions of interferencefree overlap models, as described in the Appendix. These predicted interaction sizes, shown in Table 1, are consistent with the observed effect of $51 \pm 36 \mathrm{msec}$. Thus, we conclude that the data are compatible with interferencefree overlap models.

Two additional significant effects were obtained in the analysis of RT in this and subsequent experiments. The subjects consistently responded faster to normal stimuli than to mirror-image stimuli $(p<.05)$, as is commonly found (e.g., Cooper \& Shepard, 1973). Furthermore, this effect was reliably larger when the shape discrimination was difficult than when it was easy (in Experiments $1-3, p<.05$ ).

\section{Discussion}

The significant underadditive interaction between orientation and discriminability indicates that there was temporal overlap of shape discrimination and mental rotation in this experiment, contrary to the prediction of discrete models. Furthermore, the amount of underadditivity was consistent with the predictions of models al- 


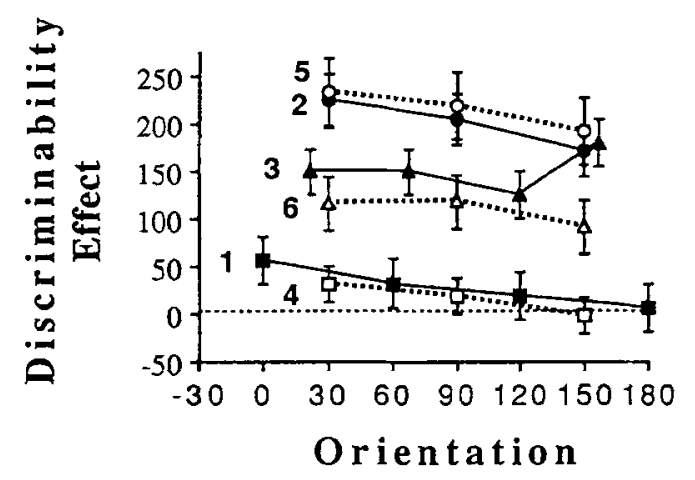

Figure 4. Mean discriminability effect, and the $95 \%$ confidence interval around it, as a function of orientation for Experiments 1-6. The solid lines correspond to the experiments involving shape discriminations (Experiments 1-3), and the dashed lines correspond to the experiments involving color discriminations (Experiments 4-6).

lowing interference-free overlap. Thus, we may tentatively conclude that mental rotation and discrimination operated simultaneously and without any mutual interference in this task.

The overall discriminability effect was not statistically reliable. This does not vitiate our conclusions, however, because the significant underadditive interaction of discriminability with orientation clearly demonstrates that the discriminability manipulation was effective. One explanation of the small main effect of discriminability is that it was obscured by the temporal overlap of shape discrimination with mental rotation. To investigate this idea, we tested 16 subjects in a control experiment using the same stimuli but requiring responses only to stimulus identity, thereby obviating the need for mental rotation. Consistent with this explanation, a reliable discriminability effect of $63 \pm 27 \mathrm{msec}$ was obtained.

Discrete models could be reconciled with the underadditive interaction between orientation and discriminability if subjects were able to rotate the stimuli more quickly in the difficult discrimination condition than in the easy discrimination condition. This alternative explanation seems unlikely because the same letters were used in both conditions. Only the letter pairings changed across conditions, and it is not clear why the rate of rotation of a given image would depend on the other images that might have been formed on a given trial. Moreover, the results of Experiments 4-6 present even more severe difficulties for this alternative explanation, as will be seen.

Another alternative explanation of these results is that stimulus orientation affects not only the required degree of mental rotation but also the difficulty of the discrimination. If the perceptual discrimination is easier for upside-down stimuli, then an underadditive interaction between orientation and discriminability could occur. This alternative explanation seems unlikely because orientation typically has little or no effect on naming latency when a small set of stimuli are used repeatedly (e.g., Corballis, Zbrodoff, Shetzer, \& Butler, 1978; White,
1980; but also see Jolicoeur \& Landau, 1984). Furthermore, even if stimulus orientation did have an effect on discriminability, one would expect discriminations to be more, not less, difficult for upside-down stimuli.

Nonetheless, we tested this explanation in the identification-only control experiment mentioned above. Subjects $(N=16)$ were shown the same stimuli as in Experiment 1 but were instructed simply to press a response key corresponding to the letter name (i.e., ignoring orientation and version). Consistent with previous findings, there was no effect of stimulus orientation $[F(3,42)<1)$; mean RTs were $552,557,551$, and $550 \mathrm{msec}$ at $0^{\circ}, 60^{\circ}, 120^{\circ}$, and $180^{\circ}$, respectively. Furthermore, the discriminability effect for upside-down stimuli was actually $8 \pm 21 \mathrm{msec}$ greater for upsidedown stimuli than for upright stimuli. Thus, there is no support for this alternative explanation of the observed underadditivity between orientation and discriminability.

Another possible explanation is that the underadditivity between orientation and discriminability is due to overlap of perception with processes that precede mental rotation. If these prerotation operations, such as determining the direction of rotation, are sensitive to stimulus orientation and operate simultaneously with shape discrimination, then underadditivity might occur, for the same reason that overlap of the actual rotation process with perception causes underadditivity. To test this hypothesis, we ran a further control experiment, using the stimuli of Experiment 1, in which subjects were to identify the stimuli and determine their orientation. Subjects $(N=16)$ responded to letter identity by pressing one of two response keys, but only if the letter appeared in the target orientation. During some blocks, the subjects were instructed to respond only to letters that were more upright than upside-down $\left(300^{\circ}, 0^{\circ}\right.$, and $60^{\circ}$ ), and, during other blocks, the subjects were instructed to respond to stimuli that were more upsidedown than upright $\left(120^{\circ}, 180^{\circ}\right.$, and $\left.240^{\circ}\right)$, with block order counterbalanced across subjects. The effects of discriminability on the time to make the identity judgments were $55,55,53$, and $49 \mathrm{msec}$ in the $0^{\circ}, 60^{\circ}, 120^{\circ}$, and $180^{\circ}$ conditions. Comparing the upright and upsidedown conditions only, this experiment yielded only a small $(6 \pm 54 \mathrm{msec})$ interaction between orientation and discriminability in the direction of underadditivity, and, therefore, this control experiment provides no support for this alternative explanation. However, because these data are somewhat noisy, we cannot say conclusively that the underadditivity observed in Experiment 1 was not due in part to the overlap of perceptual discrimination with prerotation processes.

\section{EXPERIMENT 2}

The results of Experiment 1 indicate that mental rotation can begin before shape discrimination has been completed. While the results are consistent with models allowing interference-free overlap between shape discrimination and mental rotation, the reduction of the dis- 


\section{True}

\section{Distorted}
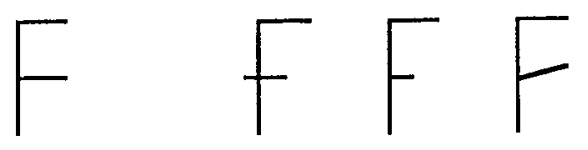

0
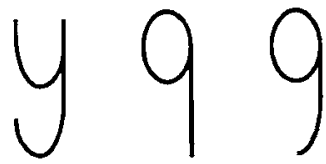

Figure 5. Examples of true and distorted stimuli used in Experiment 2 (displayed in upright positions).

criminability effect for upside-down stimuli was not very dramatic because the effect was not very large to begin with. Clearly, the success of the interference-free overlap model would be more impressive if a large discriminability effect disappeared for upside-down stimuli. This should be possible, assuming mental rotation and perception overlap without interference, because the hundreds of milliseconds needed for mental rotation allow enough time to conceal quite a large discriminability effect. Experiment 2 was thus designed as a more stringent test of interference-free overlap models, with stimuli chosen to require finer perceptual discriminations in the difficult discrimination condition. With a greater discriminability effect, interference-free overlap models predict greater underadditivity. Of course, discrete models still predict additivity.

In order to require finer perceptual discriminations, it was necessary to make the stimuli more visually similar to each other. Unfortunately, the English alphabet does not provide many pairs of letters that are highly visually similar to each other, visually dissimilar to other letters, and sufficiently nonsymmetric for use in an experiment involving mirror/normal judgments. Rather than create novel stimuli that might not have easily identifiable normal and mirror-image versions because of their unfamiliarity, we chose to use "true" and "distorted" versions of familiar stimuli (letters) and require subjects to make a true/distorted discrimination.

The true letters used in the experiment were an uppercase $F$ and a lowercase $g$. Three distorted letters were formed from each true letter by removing or displacing a line segment. Examples of these stimuli in their upright orientations are shown in Figure 5. In the difficult discrimination blocks, the subjects discriminated between true and distorted images of the same letter (e.g., $F$ vs. distorted $F$ s). In the easy discrimination blocks, the subjects discriminated between the true image of one letter and the distorted images of the other letter (e.g., $F$ vs. distorted gs).

\section{Method}

Except as noted below, the methods were identical to those of Experiment 1.
Subjects. Twenty new subjects participated in Experiment 2. Five subjects failed to meet our criterion of $80 \%$ correct and so were replaced.

Stimuli. The stimuli used in Experiment 2 were the true and distorted letters shown in Figure 5. Each letter appeared as either a normal or a mirror image and in one of six different orientations $\left(30^{\circ}, 90^{\circ}, 150^{\circ}, 210^{\circ}, 270^{\circ}\right.$, or $330^{\circ}$ from vertical $)$.

Procedure. The subjects were instructed to determine whether the stimulus letter was true or distorted and whether it was a normal or mirror-image version. Prior to beginning the experiment, the subjects were allowed to view examples of true and distorted letters until they were comfortable with the distinction. The S-R mapping was analogous to that of Experiment 1 : true/normal and distorted/mirror stimuli were assigned to one response key, whereas true/mirror and distorted/normal stimuli were assigned to the other response key.

There were eight blocks of 72 trials. Ten practice trials were given before the first block, and 6 warm-up trials were given before each subsequent block. Half of the subjects completed two difficult blocks followed by two easy blocks and so on, whereas the reverse order was used for the other half of the subjects. The true letter chosen in the first block was $F$ for half of the subjects and $g$ for the other half, and alternated thereafter.

\section{Results}

Mean RTs and error rates are shown in Figure 6 as a function of stimulus orientation and discriminability. The large effects of stimulus orientation on RT were again reliable $[F(2,36)=80.10, p<.001]$. This experiment also produced a large overall discriminability effect of $199 \pm 56 \mathrm{msec}$. As shown in Figure 4, the discriminability effect decreased monotonically across orientations, indicating an underadditive interaction between orientation and discriminability. The decrease in
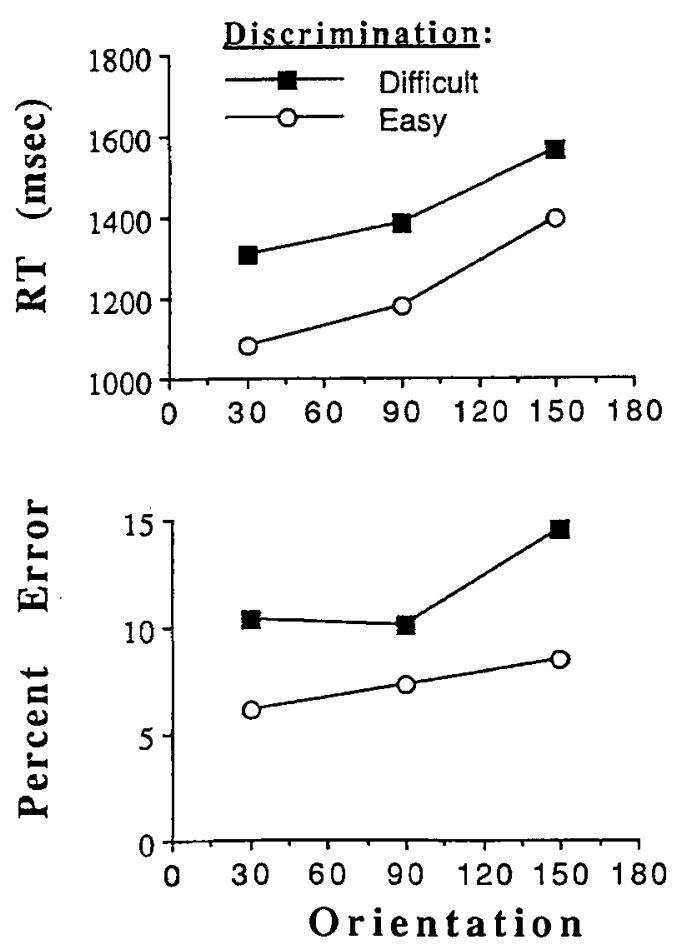

Figure 6. Mean reaction time and percent error for Experiment 2 as a function of discrimination difficulty and orientation. 
the discriminability effect between the most upright and upside-down orientations was $52 \pm 39 \mathrm{msec}$. Furthermore, a linear trend analysis revealed a reliable overall orientation $\times$ discriminability interaction on $\operatorname{RTs}[F(1,18)$ $=5.71, p<.05]$. Although the orientation $\times$ discriminability interaction on error rates was not significant $[F(2,36)=3.05, .05<p<.10]$, it should be noted that the trend is overadditive, raising the possibility that the underadditive interaction on RT was due to a speed-accuracy tradeoff.

In this experiment, the discriminability effects were 224 and $172 \mathrm{msec}$ for nearly upright and nearly upsidedown stimuli, respectively, yielding an underadditive interaction of $52 \pm 39 \mathrm{msec}$. Because the rotation of nearly upside-down stimuli took approximately $311 \mathrm{msec}$ (see Figure 6), however, intuition suggests that interferencefree overlap models would predict a much larger reduction of the discriminability effect for upside-down stimuli. As described in the Appendix, computer simulations confirmed this intuition: we failed to find any reasonable parameter estimates for an interference-free overlap model that would predict an underadditive interaction of less than $113 \mathrm{msec}$ (see Table 1). Because the observed underadditivity was clearly smaller than this, even after allowing for sampling error, these results provide evidence against interference-free overlap models.

\section{Discussion}

The significant underadditive interaction between orientation and discriminability suggests that mental rotation overlapped with shape discrimination, supporting the results of Experiment 1; however, this conclusion must be qualified by noting that some or all of this effect may have been due to a speed-accuracy tradeoff. In any case, the observed underadditivity was significantly less than predicted by models allowing interference-free overlap. These results clearly contradict interferencefree overlap models and support partial overlap models.

\section{EXPERIMENT 3}

Although the results of Experiment 2 were inconsistent with models allowing interference-free temporal overlap, that experiment employed a challenging S-R mapping. It is conceivable that the requirement to rehearse this difficult $\mathrm{S}-\mathrm{R}$ mapping could have interfered with the subjects' ability to overlap shape discrimination and mental rotation, possibly due to capacity limitations (e.g., Kahneman, 1973) or outcome conflict (e.g., Navon \& Miller, 1987). The purpose of Experiment 3 was to replicate Experiment 2 with a simplified S-R mapping and thereby determine if the failure of the interferencefree overlap model - which accounted successfully for the results of Experiment 1-was somehow due to the overall difficulty of the task.

Stimuli in Experiment 3 were chosen such that letter identity was implicitly required in order to perform the mirror/normal judgment. This simplified the required $\mathrm{S}-\mathrm{R}$ mapping, because an explicit response to identity
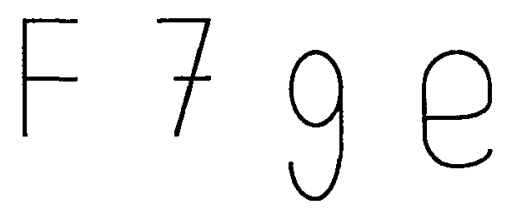

Figure 7. Stimuli used in Experiment 3 (displayed in upright positions).

was no longer required. The subjects simply pressed one key for normal versions and another key for mirrorimage versions. This was accomplished by using visually similar pairs of alphanumeric characters-namely $F, 7$ and $e, g$-that, in the fonts used, were approximate mirror images of one another, as shown in Figure 7. With such stimuli, it is necessary to identify the letter before determining whether it is a normal or a mirror image, so we can assume that the subjects identified the letters even without explicit instructions to do so. Note, however, that it was possible, at least in principle, for the subjects to determine the top of the letters and start rotating before the letters had been completely identified.

\section{Method}

Except where noted below, the methods were identical to those of Experiment 1.

Subjects. Thirty-two new subjects participated in the experiment in partial fulfillment of a course requirement or for \$6. Four subjects failed to meet our criterion of $80 \%$ correct and so were replaced.

Stimuli. The stimuli were the letters $e, g, F$, the number 7 (see Figure 7), and their mirror images, drawn in light gray on a black background. The stimuli appeared in one of eight possible orientations spaced $45^{\circ}$ apart $\left(22^{\circ}, 67^{\circ}, 112^{\circ}\right.$, or $157^{\circ}$ clockwise and counterclockwise from vertical). Because we avoided horizontal and vertical orientations, the letters appeared roughly equally jagged at all orientations.

Procedure. The discriminability manipulation and the assignment of stimuli to the easy and difficult blocks was identical to that of Experiment 1, except that a different set of stimulus letters $(F, 7$ and $g, e$ ) was used. Half of the subjects pressed the " $z$ " key in response to normal images and the "" key in response to mirror images; for the other half, these assignments were reversed.

Each session lasted approximately $40 \mathrm{~min}$ and consisted of four blocks of 160 trials. The first block was preceded by 12 randomly selected practice trials, the second block by 6 warm-up trials, and subsequent blocks by 2 warm-up trials.

\section{Results}

The mean RTs and error rates for Experiment 3 are displayed in Figure 8 as a function of orientation and discrimination difficulty. RT analyses again revealed reliable effects of orientation $[F(3,90)=121.61, p<.001]$, and the mean discriminability effect in this experiment was $151 \pm 41 \mathrm{msec}$. Overall, the orientation $\times$ discriminability interaction was significant for $\operatorname{RT}[F(3,90)=$ $3.40, p<.05]$ and marginally significant for error rates $[F(3,90)=2.72, p>.05]$. However, as can be seen in Figure 4 , the interactive effect on RT was not monotonic across orientations, and, considering only the most extreme orientations, there was a nonsignificant overaddi- 

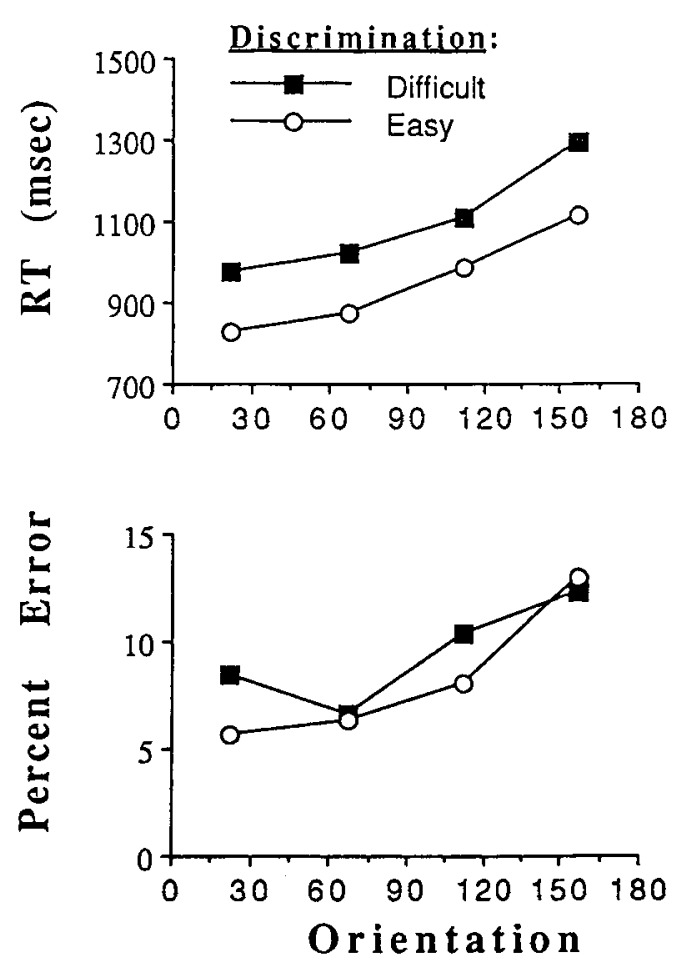

Figure 8. Mean reaction time and percent error for Experiment 3 as a function of discrimination difficulty and orientation.

tive interaction of $30 \pm 34 \mathrm{msec}$. The overall pattern of the orientation $\times$ discriminability interaction is not predicted by any of the models. This pattern may have been caused by slight differences in stimulus discriminabilities at the different orientations, due to the use of a limited-precision pixel display. Furthermore, the discriminability effect on error rates dropped noticeably at $157^{\circ}$, where the discriminability effect on RT was largest, so a speed-accuracy tradeoff may have been partially responsible for this pattern of results. Overall, however, the results seem more consistent with discrete models than with continuous models, since the overall interaction was certainly not underadditive. Most importantly, the results clearly argue against models allowing interference-free overlap, which predict large underadditive interactions (see Table 1).

\section{Discussion}

The main purpose of Experiment 3 was to determine if the failure of the interference-free overlap model in Experiment 2 could be attributed to the complexity of the $S-R$ mapping or the overall difficulty of the experiment. A simplified S-R mapping was used that successfully reduced overall RTs by several hundred milliseconds. However, the results were again inconsistent with models allowing interference-free temporal overlap. Indeed, the lack of underadditivity is most consistent with discrete models. It is not certain exactly what caused the significant nonmonotonic interaction of discriminability and orientation in this experiment, but the results are clearly inconsistent with interference-free overlap models in any case.

\section{DISCUSSION OF EXPERIMENTS 1-3}

Contrary to discrete models, Experiments 1 and 2 yielded underadditive interactions on RT between orientation and discriminability. It appears, therefore, that the perceptual system, in some cases, transmits to the mental rotation process partial information (i.e., enough information for rotation to begin) before shape discrimination has been completed. On the other hand, the amounts of underadditivity observed in Experiments 2 and 3 were much too small to be compatible with models postulating interference-free overlap between mental rotation and perception. Thus, it appears that fully continuous models can also be rejected and that the correct model must allow some type of partial overlap. Before attempting to specify the partial overlap model in more detail, we will present further evidence concerning the temporal overlap of mental rotation and color discrimination.

\section{EXPERIMENT 4}

One possible explanation for the lack of interferencefree overlap in Experiments 1-3 is that different operations involving shape (i.e., fine shape discriminations and rotation) interfere with one another when they are carried out simultaneously. In order to investigate this possibility, Experiments 4-6 tested for temporal overlap of mental rotation with color, rather than shape, discrimination. Because mental rotation and color discrimination clearly operate upon distinct stimulus codes (color vs. shape), any dimension-specific interference should be greatly reduced or eliminated. The primary purpose of Experiment 4 was to determine whether mental rotation can overlap with color discrimination when the discriminability effect is relatively small. Experiments 5 and 6 used much larger discriminability effects.

The design of Experiment 4 was analogous to that of Experiment 1 . The subjects viewed colored letters and based their responses on both color and mirror/normal version. The subjects discriminated between green and yellow letters in the difficult discrimination blocks and between red and white letters in the easy discrimination blocks.

\section{Method}

Subjects. Sixty-four new subjects with normal color vision participated in the experiment in partial fulfillment of a course requirement. Five subjects failed to meet our criterion of $80 \%$ correct and were therefore replaced.

Stimuli. The stimuli were the uppercase letters $F, R, J$, the number 7 , and their mirror images. These stimuli appeared in one of six possible orientations $\left(30^{\circ}, 90^{\circ}, 150^{\circ}, 210^{\circ}, 270^{\circ}\right.$, or $330^{\circ}$ from vertical). The four colors used in the experiment were red, white, yellow, and green, corresponding to IBM enhanced graphics adapter codes $4,15,14$, and 10 , respectively. All stimuli were displayed against a dark background. 
Procedure. At the beginning of the experiment, the subjects were told that they would be rewarded between $\$ 0.50$ and $\$ 2$ for participating, contingent upon their speed and accuracy. They were instructed to quickly determine both the color and the version of the letter on each trial. Stimuli were mapped to responses in a manner analogous to that of Experiments 1 and 2: the normal letters of one color and the mirror-image letters of the other color were assigned to one response key, whereas mirror-image letters of the first color and normal letters of the second color were assigned to the remaining response key.

Stimuli appeared in red or white during the easy discrimination blocks and in green or yellow during the difficult blocks. The computer warned the subjects each time a new block began and informed them which colors would be used. Half of the subjects began the experiment with an easy block, and half began with a difficult block. Block type then alternated until all four blocks had been completed.

For each trial within a block, one of the four stimuli $(F, R, J$, and $7)$ was chosen randomly. The color, orientation, and version (normal or mirror image) of the stimuli were also chosen randomly, with the restriction that each color/orientation/version combination occur equally often within a block of trials.

Each session lasted approximately $45 \mathrm{~min}$ and consisted of four blocks of 144 trials. The first block was preceded by 20 randomly selected practice trials; subsequent blocks were preceded by 4 randomly selected warm-up trials.

\section{Results}

The mean RTs and error rates for Experiment 4 are displayed in Figure 9 as a function of orientation and discrimination difficulty. The large effects of orientation on RT were again highly reliable $[F(2,124)=360.12, p<$ $.001]$. However, the overall discriminability effect in this experiment was only $16 \pm 26 \mathrm{msec}$. Figure 4 shows the discriminability effect as a function of orientation; the negative slope indicates an underadditive interaction between orientation and discriminability. Comparing data from the upright and upside-down orientations, this interaction was $33 \pm 27 \mathrm{msec}$. Furthermore, the linear contrast of this interaction on RT was significant $[F(1,62)=$ $5.36, p<.05]$. The reliable underadditive interaction between discrimination difficulty and orientation contradicts discrete models but is consistent with interferencefree and partial overlap models.

The discriminability effect in this experiment virtually disappeared for upside-down stimuli, just as predicted by models allowing interference-free overlap. As shown in Table 1, interference-free overlap models can generate underadditivities of the size observed in this experiment, so the data from this experiment are consistent with such models.

\section{Discussion}

The significant underadditive interaction between orientation and discriminability provides further evidence against strictly discrete models, in which perceptual discriminations and mental rotation are performed serially. Furthermore, as in Experiment 1, the amount of underadditivity is consistent with the predictions of "models postulating" interference-free overlap of color discrimination and mental rotation.
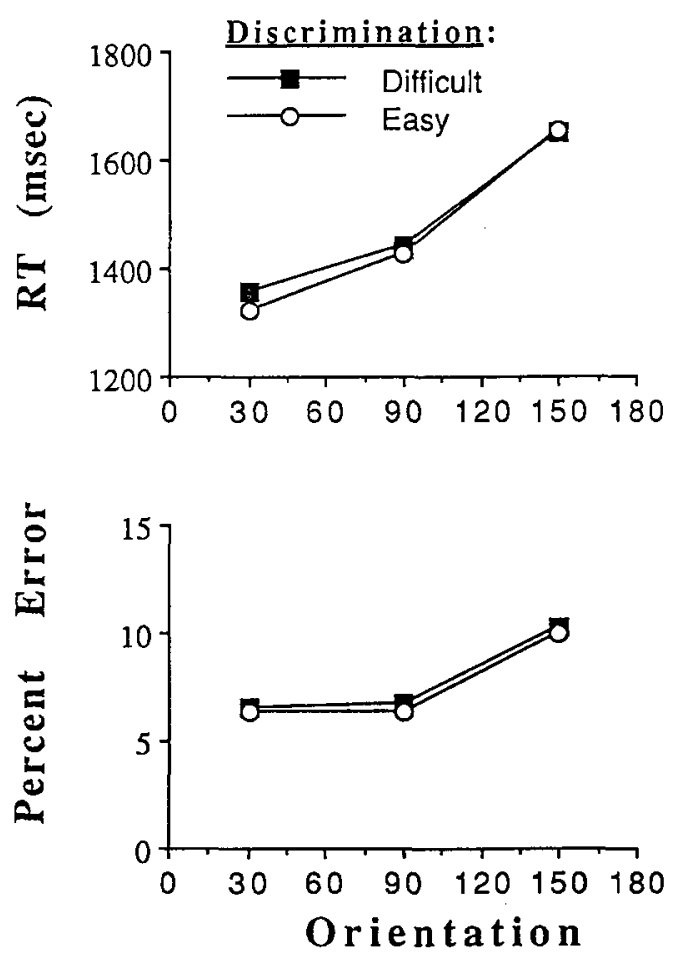

Figure 9. Mean reaction time and percent error for Experiment 4 as a function of discrimination difficulty and orientation.

In Experiment 1, we mentioned the possibility that underadditivity between orientation and discriminability could result if the subjects were able to rotate the letters more quickly in the difficult discrimination blocks. This alternative explanation cannot account for the results of the present experiment because the only difference between the easy and difficult blocks was the color of the stimuli, which clearly should have no effect on the rate of rotation. Because the orientation $\times$ discriminability interaction observed in this experiment bears a striking resemblance to that of Experiment 1 (see Figure 4), both in terms of overall size and the pattern across orientations, it also appears unlikely that the rotation speed-up hypothesis accounted for the results of Experiment 1.

As was the case with Experiment 1, the overall discriminability effect did not reach significance. This is not cause for concern, however, because a control experiment, in which the subjects responded to stimulus color but not mirror/normal version, indicated a reliable discriminability effect of $51 \pm 23 \mathrm{msec}$. Furthermore, the significant orientation $\times$ discriminability interaction clearly indicates that the discriminability manipulation had an effect.

These initial results suggest that mental rotation begins before perception has finished and can overlap with color discrimination - possibly without interferenceat least when the color discrimination is not too difficult. The purpose of Experiments 5 and 6 was to conduct a more challenging test of the interference-free overlap model by increasing the size of the discriminability effect. 


\section{EXPERIMENT 5}

To make the color discrimination more challenging, we increased the number of colors within a block from two to three. In both the easy and difficult blocks, the subjects discriminated red from non-red stimuli. In the easy block, the subjects discriminated red letters from blue and green letters; in the difficult block, the subjects discriminated red letters from purple and orange-red letters.

\section{Method}

Except where noted, the methods were identical to those of Experiment 4.

Subjects. Thirty-two new subjects with normal color vision participated in the experiment in partial fulfillment of a course requirement.

Stimuli. The stimuli were identical, except in color, to those of Experiment 4. The five colors used in the experiment were red, orange-red, purple, blue, and green, corresponding to IBM enhanced graphics adapter codes $4,6,5,9$, and 10 , respectively.

Procedure. The subjects were told that they would need to determine both the color (red vs. non-red) and the version of the letter. The response mapping was similar to that used in Experiment 4. The subjects pressed one key if the letter was red/normal or non$\mathrm{red} / \mathrm{mirror}$ and another key if the letter was red/mirror or non$\mathrm{red} /$ normal.

During the easy discrimination blocks, stimuli appeared in red, green, or blue; during the difficult discrimination blocks, stimuli appeared in red, orange-red, or purple. The probability of a red letter was .5; the probability of each of the two non-red colors was .25 . The computer warned the subjects each time a new block began and displayed the colors to be used.

\section{Results}

The mean RTs and error rates for Experiment 5 are displayed in Figure 10 as a function of orientation and discrimination difficulty. RT analyses again revealed large effects of orientation $[F(2,60)=81.63, p<.001]$. The overall discriminability effect was $216 \pm 68 \mathrm{msec}$. Figure 4 shows that the discriminability effect on RT once again decreased monotonically across orientations, suggesting an underadditive interaction between orientation and discriminability. However, the decrease of 41 $\pm 50 \mathrm{msec}$, measured between the most upright and upside-down orientations, is not statistically significant. The linear component of the interaction also failed to reach significance $[F(1,30)=2.65, p>.2]$. Thus, this experiment, by itself, does not provide clear evidence against discrete models.

The amount of underadditivity observed in this experiment $(41 \pm 50 \mathrm{msec})$ is very small in comparison with the size of the discriminability effect $(216 \mathrm{msec})$. Therefore, the data do not appear to be consistent with interference-free temporal overlap. Indeed, none of the predicted underadditivities (see Table 1) fall within the range of observed values, confirming that the interferencefree overlap model is not consistent with these data.

\section{Discussion}

Although we again observed a small underadditive trend in the orientation $\times$ discriminability interaction, it
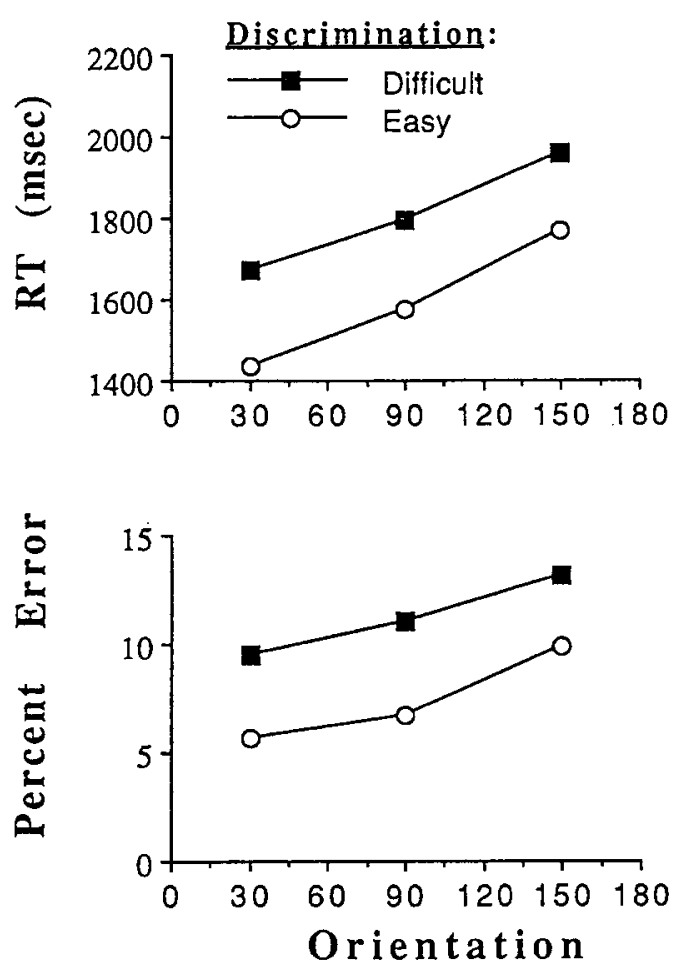

Figure 10. Mean reaction time and percent error for Experiment 5 as a function of discrimination difficulty and orientation.

did not reach significance, and so discrete models cannot be rejected for the present experiment. Because the main effect of discriminability was quite large, however, the absence of a large underadditivity does allow us to reject interference-free overlap models, although, of course, it is still possible that there is some small amount of partial overlap. Because the failure of the interferencefree overlap model in Experiments 2 and 3 has now also been observed when the perceptual task involves color discrimination, this failure cannot be attributed solely to the similarity of the representations used by shape discrimination and mental rotation.

\section{EXPERIMENT 6}

Experiment 5 provided evidence against interferencefree temporal overlap between color discrimination and mental rotation. Experiment 6 was an attempt to replicate this result with a less complicated response mapping. We employed a choice/no-go task, which is like a go/no-go task except subjects can make one of two responses on the go trials. That the task was indeed much easier than that used in Experiment 5 is demonstrated by a decrease of approximately $300 \mathrm{msec}$ in overall RT.

\section{Method}

Except where noted, the methods were identical to those of Experiment 5.

Subjects. Thirty-two new subjects with normal color vision participated in the experiment in partial fulfillment of a course requirement. 
Procedure. The subjects were instructed to determine both the color (red vs. non-red) and the version of the letter. The subjects were asked to respond to normal images but not to mirror images. When the stimulus letter was a normal image, the subjects pressed one key if the letter was displayed in red and another key if it was not displayed in red. On no-go trials, the mirror-image stimulus letters were terminated after $3 \mathrm{sec}$; otherwise, the stimulus presentation sequence was identical to that of Experiment 5.

\section{Results}

Go trials. Mean RTs and error rates for go trials are displayed in Figure 11 as a function of orientation and discrimination difficulty. On go trials, the computer waited until the subjects made a response, so the subjects never failed to respond. Errors, therefore, represent incorrect key presses only. ${ }^{6} \mathrm{RT}$ analyses revealed reliable effects of orientation $[F(2,60)=126.72, p<.001]$, and the overall discriminability effect was $108 \pm 58 \mathrm{msec}$. Figure 4 shows that the discriminability effect on RT again decreased monotonically with increasing orientation. The decrease of $24 \pm 39 \mathrm{msec}$ between the two most extreme orientations, however, is not statistically significant. The linear interaction contrast also failed to reach significance $[F(1,30)=2.65, p>.1]$. Therefore, we do not have enough evidence to rule out discrete models.

The observed interactions seem much too small to be consistent with interference-free temporal overlap. In fact, all of the interference-free overlap models examined predicted larger underadditivities than those observed (see Table 1), so we conclude that the data are incompatible with such models.
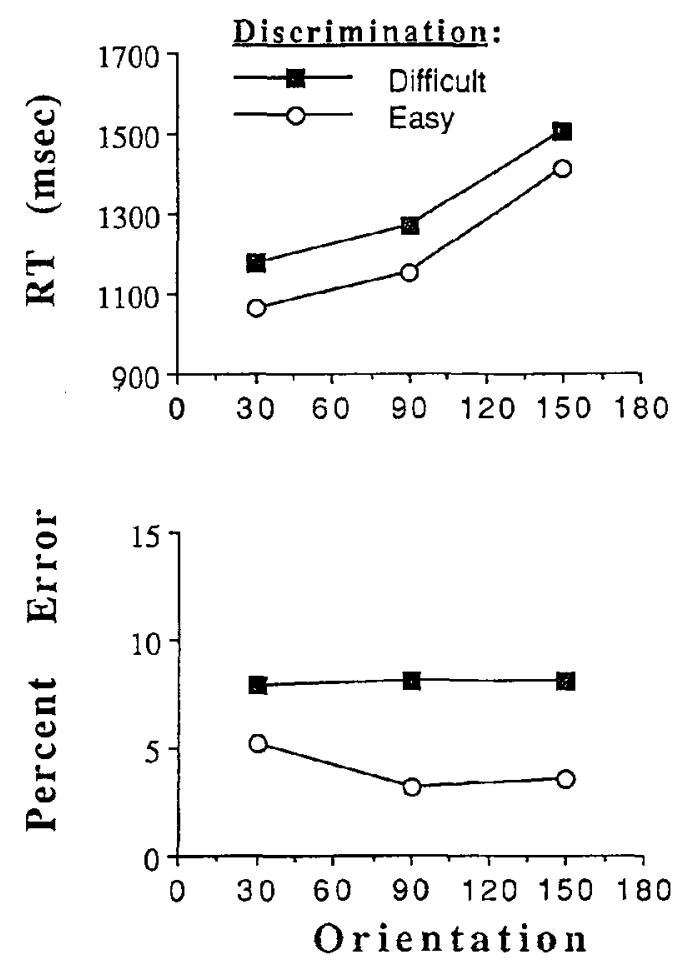

Figure 11. Mean reaction time and percent error for Experiment 6 as a function of discrimination difficulty and orientation.
No-go trials. The subjects responded to no-go stimuli (i.e., mirror images) on $6.1 \%$ of the trials. These errors occurred more frequently for upside-down stimuli than for upright stimuli $[F(2,60)=32.27, p<.001]$.

\section{Discussion}

Overall, the results resemble those of Experiment 5. As in that experiment, the orientation $\times$ discriminability interaction showed a small underadditive trend, but the trend was not statistically reliable. Furthermore, the interaction was again too small to be consistent with models allowing interference-free overlap between color discrimination and mental rotation. Apparently, then, the difficulty of the response mapping used in Experiment 5 was not responsible for the lack of interference-free overlap between color discrimination and mental rotation. Note that a similar conclusion was reached in Experiment 3 regarding the lack of interference-free overlap between shape discrimination and mental rotation.

The size of the discriminability $\times$ orientation interaction observed in this experiment was similar to that of Experiment 5, although the overall discriminability effect was only half that observed in Experiment 5 (108 vs. $216 \mathrm{msec}$ ). This was surprising because the experiments required identical color discriminations. The apparent discrepancy in the size of the discriminability effect might be due to differences between groups of subjects or perhaps to unintended changes in the contrast or intensity of the displays. More likely, however, the difference is due to the nature of the S-R mappings. A pilot experiment in which the subjects responded only to the color of the stimuli (red vs. non-red), and therefore involved a simple S-R mapping, produced a discriminability effect of $105 \mathrm{msec}$, which lies very close to the effect size observed in the present experiment $(108 \mathrm{msec})$. Apparently, the color discrimination is slowed when subjects are faced with a difficult S-R mapping, perhaps because subjects cannot optimally prepare for the color discrimination when they must also prepare for such a difficult mapping. Seemingly related effects have been observed in dual-task experiments (e.g., Gottsdanker, 1979; Pashler, 1984). Gottsdanker, for instance, found that performance on a first task is worse when subjects expect another task to follow immediately, even on trials in which the stimulus for the second task never comes.

\section{GENERAL DISCUSSION}

Using an additivity/underadditivity diagnostic, we sought to determine whether mental rotation overlaps with shape and color discriminations. Whereas discrete stage models predict additive relations between stimulus orientation and discriminability in the prese-"- experiments, models allowing temporal overlap preaict underadditive interactions.

Overall, the results exhibited a remarkably consistent trend toward underadditivity. This trend is shown in Figure 4 , which displays discriminability effects as a function of stimulus orientation for all six experiments. 
Discriminability effects consistently diminished with increasing orientation (in degrees from upright), suggesting that there is at least some temporal overlap of mental rotation and discrimination. The only experiment that did not show a monotonic underadditive trend was Experiment 3 , in which the discriminability effect was greatest when the stimulus was nearly upside-down. So, although the underadditivity is not large and the evidence of overlap from several of the individual experiments could perhaps be called into question, the consistency of the underadditivity suggests that it is genuine. Thus, we conclude that the data as a whole indicate overlap of discrimination and mental rotation.

Surprisingly, the amount of underadditivity did not appear to depend upon the magnitude of the discriminability effect. Even though the discriminability effect for near-upright stimuli varied from approximately 31 to $234 \mathrm{msec}$, the reduction in the effect across orientations remained small and approximately constant, as reflected by the similar slopes of all lines in Figure 4 . Note that this qualitative finding is inconsistent with interferencefree overlap models, which predict that the size of the underadditivity should increase with the size of the discriminability main effect.

The amounts of underadditivity in Experiments 1-3, which required shape discriminations, were roughly equal to the amounts of underadditivity in Experiments 4-6, which required color discriminations (compare the solid and dashed lines in Figure 4). This suggests that the amount of overlap is not influenced by the similarity of the operations needed for mental rotation and the required discrimination nor by the similarity of the types of information being manipulated.

Because the underadditivity of orientation and discriminability contradicts fully discrete models of information processing, the present results suggest that the perceptual system transmits asynchronously several outputs about a single stimulus to later stages. That is, the perceptual process appears to transmit some outputs (i.e., an image used by the mental rotation process) before other outputs (i.e., color and identity codes) have been produced, at least on some trials. The results do not indicate, however, whether this early output is itself transmitted in discrete chunks or in a series of small (i.e., continuous) transmissions. The present evidence of temporal overlap is consistent with a number of studies that have demonstrated temporal overlap of perception and response preparation (see Miller, 1988, for a review; see Miller \& Hackley, 1992, Miller, Riehle, \& Requin, 1992, and Osman et al., 1992, for more recent examples). The present results extend these findings by showing that mental rotation also can overlap with perception.

Although the results, overall, exhibited sufficient underadditivity to reject strictly discrete models of information processing, the amount of underadditivity tended to be fairly small, even when the overall discriminability effect was very large (see Figure 4). This finding is incompatible with models allowing interference-free overlap of perception and mental rotation. Because mental rotation of upside-down stimuli takes a very long time, models with interference-free overlap predict that even a large discriminability effect should all but disappear for these stimuli. Thus, interference-free overlap predicts large reductions in discriminability effects across orientations, contrary to the present observations. This apparent contradiction was verified by computing the predicted underadditivity for a wide range of models allowing interference-free overlap, as described in the Appendix. In four of the six experiments, the observed underadditivity was less (even allowing for sampling error) than that predicted by any interference-free overlap model that we examined (see Table 1). The results of these experiments, then, appear to be incompatible with models allowing interference-free temporal overlap, including both fully continuous models and the ADC model (Miller, 1982). The latter model predicts discrete processing or interferencefree overlap, depending on whether distinct codes are used for mental rotation and perceptual discriminations, but, without further modification, cannot be reconciled with evidence of partial overlap.

Any satisfactory model must be able to explain the presence of partial, but not interference-free, temporal overlap between perception and mental rotation in the present experiments. One possibility is that a probability mixture is responsible for the results, with discrete processing on some trials and interference-free overlap on others. Similarly, some subjects might transmit information between stages discretely, while others process continuously. To account for the fact that the underadditivity did not depend on the size of the discriminability effect, these explanations would require the assumption that the mixture probabilities depended on discriminability in just such a way as to leave constant the average underadditive effect on mean RT. In the absence of any direct evidence supporting this model, it seems unlikely because it requires such fortuitous changes in mixture probabilities.

Two more attractive explanations are based on the idea that mental rotation and perception interfere with one another when they operate concurrently. First, mental rotation and perceptual discrimination might indeed be carried out at the same time, but substantial interference might cause each one to proceed more slowly than it would in isolation. Such interference might result from capacity sharing (e.g., Kahneman, 1973) or from outcome conflicts (e.g., Navon \& Miller, 1987), to name two possibilities. With the proper amount of interference, such models could presumably predict the amount of underadditivity observed in these experiments.

Second, it is possible that mental rotation can overlap with only a subset of perceptual operations. We will refer to this as the central bottleneck explanation (e.g., Pashler, 1984; Welford, 1952), because a plausible version of it is one in which both mental rotation and certain perceptual operations require access to a singlechannel central mechanism. According to this hypothesis, mental rotation overlaps with initial perceptual encoding, which does not require the bottleneck, but 
cannot be carried out in parallel with fine perceptual discriminations, which do require the bottleneck.

We are not aware of any published attempts to demonstrate interference between mental rotation and perception, which would clearly support our explanation of partial overlap in terms of interference. There are, however, several reports that mental rotation interferes in some way with other difficult cognitive operations, including response selection (Ruthruff, Miller, \& Lachmann, 1995), response preparation (Band \& Miller, 1994), and size-scaling operations (Bundesen, Larsen, \& Farrell, 1981). There is also evidence of interference when two different mental rotations must be performed simultaneously (Murray, Corballis, \& Campsall, 1992). Collectively, these findings support the idea that mental rotation could interfere with the simultaneous operation of another mental process. Similarly, there is evidence that difficult perceptual discriminations are subject to interference among themselves (e.g., Bonnel, Stein, \& Bertucci, 1992) and with other mental processes, such as response selection (McCann \& Johnston, 1989). Given that both mental rotation and difficult perceptual operations interfere with other difficult mental operations, it would not be surprising if they interfered with each other. The present data are unable to discriminate between the central bottleneck and capacity-limitation explanations of the apparent interference between perception and mental rotation.

\section{Concluding Remarks}

These results provide evidence for partial overlap between perception and mental rotation. Several conclusions can therefore be reached. First, the transmission of information from perception to the mental rotation process is nondiscrete; instead, a preliminary perceptual representation is passed to the mental rotation process before all task-relevant perceptual analysis (i.e., of shape or color) is finished. Second, mental rotation is able to operate during some of the operations required for perceptual discrimination. Third, interference prevents complete overlap of perceptual discrimination and mental rotation. This interference could be a result of occasional delays in the transmission of partial information or competition between simultaneous perceptual discrimination and mental rotation processes (e.g., due to a central bottleneck).

\section{REFERENCES}

BAND, G. P. H., \& Miller, J. O. (1994). Mental rotation interferes with response preparation. Unpublished manuscript.

Bonnel, A. M., Stein, J. F., \& BertuCCI, P. (1992). Does attention modulate the perception of luminance changes? Quarterly Journal of Experimental Psychology, 44A, 601-626.

Bundesen, C., Larsen, A., \& Farrell, J. E. (1981). Mental transformations of size and orientation. In J. B. Long \& A. D. Baddeley (Eds.), Attention and performance $I X$ (pp. 279-294). Hillsdale, NJ: Erlbaum.

CHocholle, R. (1945). Variation des temps de reaction auditifs en fonction de l'intensité à diverses frequences. Année Psychologique, 41, 65-124
Cohen, N. J., \& SQuire, L. R. (1980). Preserved learning and retention of pattern-analyzing skill in amnesia: Dissociation of knowing how and knowing that. Science, 210, 207-210.

Coles, M. G. H. (1989). Modern mind-brain reading: Psychophysiology, physiology, and cognition. Psychophysiology, 26, 251-269.

CoOPER, L. A., \& ShePard, R. N. (1973). Chronometric studies of the rotation of mental images. In W. G. Chase (Ed.), Visual information processing (pp. 75-176). New York: Academic Press.

Corballis, M. C., Zbrodoff, N. J., Shetzer, L. I., \& Butler, P. B. (1978). Decisions about identity and orientation of rotated letters and digits. Memory \& Cognition, 6, 98-107.

Damasio, A. R. (1985). Prosopagnosia. Trends in Neurosciences, 8, 132-135.

Egeth, H. E., \& Dagenbach, D. (1991). Parallel versus serial processing in visual search: Further evidence from subadditive effects of visual quality. Journal of Experimental Psychology: Human Perception \& Performance, 17, 551-560.

ERIKSEN, C. W., \& SchULTZ, D. W. (1979). Information processing in visual search: A continuous flow conception and experimental results. Perception \& Psychophysics, 25, 249-263.

GoTTSDANKER, R. (1979). A psychological refractory period or an unprepared period? Journal of Experimental Psychology: Human Perception \& Performance, 5, 208-215.

Greenhouse, S. W., \& Geisser, S. (1959). On methods in the analysis of profile data. Psychometrika, 24, 95-112.

JolicoeUr, P., \& LANDAU, M. J. (1984). Effects of orientation on the identification of simple visual patterns. Canadian Journal of Psychology, 38, 80-93.

KAHNEMAN, D. (1973). Attention and effort. Englewood Cliffs, NJ: Prentice-Hall.

LUCE, R. D. (1986). Response times: Their role in inferring elementary mental organization. Oxford: Oxford University Press.

McCAnn, R. S., \& Johnston, J. C. (1989, November). The locus of processing bottlenecks in the overlapping tasks paradigm. Paper presented at the meeting of the Psychonomic Society, Atlanta.

MCClELLAND, J. L. (1979). On the time relations of mental processes: A framework for analyzing processes in cascade. Psychological Review, 86, 287-330.

Meyer, D. E., Osman, A. M., Irwin, D. E., \& Yantis, S. (1988). Modern mental chronometry. Biological Psychology, 26, 3-67.

Miller, J. O. (1982). Discrete versus continuous stage models of human information processing: In search of partial output. Journal of Experimental Psychology: Human Perception \& Performance, $\mathbf{8}$, 273-296.

MiLLER, J. O. (1983). Can response preparation begin before stimulus recognition finishes? Journal of Experimental Psychology: Human Perception \& Performance, 9, 161-182.

MiLlER, J. O. (1987). Evidence of preliminary response preparation from a divided attention task. Journal of Experimental Psychology: Human Perception \& Performance, 13, 425-434.

MILLER, J. O. (1988). Discrete and continuous models of human information processing: Theoretical distinctions and empirical results. Acta Psychologica, 67, 191-257.

Miller, J. O. (1993). A queue-series model for reaction time, with discrete-stage and continuous-flow models as special cases. Psychological Review, 106, 702-715.

Miller, J. O., \& HaCkLey, S. A. (1992). Electrophysiological evidence for temporal overlap among contingent mental processes. Journal of Experimental Psychology: General, 121, 195-209.

Miller, J. O., Riehle, A., \& ReQuin, J. (1992). Effects of preliminary perceptual output on neuronal activity of the primary motor cortex. Journal of Experimental Psychology: Human Perception \& Performance, 18, 1121-1138.

Murray, J. E., Corballis, M. C., \& Campsall, J. (1992, November). Piece-by-piece rotation of visual image pairs. Paper presented at the meeting of the Psychonomic Society, St. Louis.

Myerson, J., Widaman, K., \& Hale, S. (1990, November). Theoretical implications of the variability of speeded information processing. Paper presented at the meeting of the Psychonomic Society, New Orleans.

Navon, D., \& Miller, J. O. (1987). Role of outcome conflict in dual- 
task interference. Journal of Experimental Psychology: Human Perception \& Performance, 13, 435-448.

Norman, D. A., \& Bobrow, D. G. (1975). On data-limited and resource-limited processes. Cognitive Psychology, 7, 44-64

Osman, A. M., Bashore, T. R., Coles, M. G. H., Donchin, E., \& MEYER, D. E. (1992). On the transmission of partial information: Inferences from movement-related brain potentials. Journal of Experimental Psychology: Human Perception \& Performance, 18, 21 7-232.

PASHLER, H. E. (1984). Processing stages in overlapping tasks: Evidence for a central bottleneck. Journal of Experimental Psychology. Human Perception \& Performance, 10, 358-377.

RABBITT, P. M. A. (1986). Models and paradigms in the study of stress effects. In G. R. J. Hockey, A. W. K. Gaillard, \& M. G. H. Coles (Eds.), Energetics and human information processing (pp. 155174). Dordrecht, the Netherlands: Martinus Nijhoff.

Ruthruff, E. D., Miller, J. O., \& Lachmann, T. (1995). Does mental rotation require central mechanisms? Journal of Experimental Psychology: Human Perception \& Performance, 21, 552-570.

SANDERS, A. F. (1980). Stage analysis of reaction processes. In G. E. Stelmach \& J. Requin (Eds.), Tutorials in motor behavior (pp. 331 354). Amsterdam: North-Holland.

SANDERS, A. F. (1983). Towards a model of stress and human performance. Acta Psychologica, 53, 61-97.

SANDERS, A. F. (1990). Issues and trends in the debate on discrete vs. continuous processing of information. Acta Psychologica, 74, 123-167.

SCHWEICKERT, R., \& TOWNSEND, J. T. (1989). A trichotomy: Interactions of factors prolonging sequential and concurrent mental processes in stochastic discrete mental (PERT) networks. Journal of Mathematical Psychology, 33, 328-347.

SHAFFER, L. H. (1971). Attention in transcription skill. Quarterly Journal of Experimental Psychology, 23, 107-112.

ShePARD, R. N., \& COOPER, L. A. (1982). Mental images and their transformations. Cambridge, MA: MIT Press/Bradford Books.

Smid, H. G. O. M., Lamain, W., Hogeboom, M. M., Mulder, G., \& Mulder, L. J. M. (1991). Psychophysiological evidence for continuous information transmission between visual search and response processes. Journal of Experimental Psychology: Human Perception \& Performance, 17, 696-714.

Stanovich, K. E., \& Pachella, R. G. (1977). Encoding, stimulusresponse compatibility, and stages of processing. Journal of Experimental Psychology: Human Perception \& Performance, 3, 41 1-421.

STERNBERG, S. (1969). The discovery of processing stages: Extensions of Donders' method. Acta Psychologica, 30, 276-315.

STERNBERG, S. (1975). Memory scanning: New findings and current controversies. Quarterly Journal of Experimental Psychology, 27, $1-32$.

TownSEND, J. T., \& ASHBY, F. G. (1983). The stochastic modeling of elementary psychological processes. Cambridge: Cambridge University Press.

Welford, A. T. (1952). The "psychological refractory period" and the timing of high-speed performance-A review and a theory. British Journal of Psychology, 43, 2-19.

WHITE, M. J. (1980). Naming and categorization of tilted alphanumeric characters do not require mental rotation. Bulletin of the Psychonomic Society, 15, 153-156.

WHITT, W. (1976). Bivariate distributions with given marginals. Annals of Statistics, 4, 1280-1288.

\section{NOTES}

1. Pilot experiments indicated that the time to make these perceptual discriminations is not greatly affected by stimulus orientation, and it is therefore unlikely that such discriminations require any mental rotation.

2. Despite instructions emphasizing accuracy, a substantial proportion of subjects (roughly $15 \%$ ) in these experiments failed to achieve $80 \%$ correct. These subjects have been excluded from all analyses reported in this article. However, we have conducted additional analyses including these subjects and found negligible differences.

3 . In all of the analyses of variance reported in this article, $p$ values have been adjusted using Greenhouse and Geisser's (1959) correction where appropriate.
4. All confidence intervals reported in this article are $95 \%$ confidence intervals.

5. Specifically, we examined the linear decrease in discriminability effects as a function of the average mental rotation times for each orientation in the easy discrimination condition.

6 . When subjects initially mistake a go stimulus for a no-go stimulus, they eventually discover their error, because only go stimuli (i.e., normal versions) remain on the screen for longer than $3 \mathrm{sec}$. This may result in a small number of unusually long RTs on go trials. Accordingly, we removed any RTs longer than $3 \mathrm{sec}$. Furthermore, we have conducted additional analyses based on median RTs. The results of these analyses were similar to those reported below based on mean RTs, so there is no reason to believe that any of our findings were due to outlier-based artifacts.

\section{APPENDIX}

This Appendix describes the procedures used to compute predictions from models allowing interference-free temporal overlap between perceptual discriminations and mental rotation. Our goal was to determine how large an underadditive interaction between discriminability and orientation such models predict for Experiments 1-6, so that these predictions could be compared with the observed amounts of underadditivity.

\section{Interference-Free Overlap Models}

A general characterization of models with interference-free temporal overlap is shown in Figure Ala. The box labeled "Perception" represents those processes required to uniquely determine the shape (Experiments 1-3) or color (Experiments 4-6) of the stimulus letters. The box labeled "Mental Rotation" represents not only the process used to compensate for nonupright stimulus orientation but also the process of deciding whether the stimulus is a normal or mirror image. The random variable $T_{\mathrm{o}}$, meanwhile, represents the time required for the processes leading up to mental rotation (e.g., determining general shape and the direction of rotation). In this model, the perceptual stage begins with stimulus onset, whereas the mental rotation stage begins some time later $\left(T_{0}\right)$, and each proceeds until it is completed (at times $\mathrm{P}$ and $\mathrm{M}$, respectively). Interference-free overlap implies that each process begins at the same time and proceeds at the same rate as it would if it were performed in isolation. In other words, there is no interference between simultaneous discrimination and rotation processes, as might occur given capacity limitations or delays in information transmission between processing stages. Once both operations have finished, later processes (e.g., response selection) begin, ending in the execution of a response.

The total RT for this model is equal to the maximum of the finishing times of perception $(\mathrm{P})$ and mental rotation $(\mathrm{M})$ plus the duration of the later $(\mathrm{L})$ processes:

$$
\mathrm{RT}=\max (\mathrm{P}, \mathrm{M})+\mathrm{L} \text {. }
$$

Because the distribution of $\mathrm{L}$ is assumed to be the same across all conditions of the experiments and we are concerned solely with differences in mean RTs between conditions, we ignored $\mathrm{L}$ and simply computed $\max (\mathrm{P}, \mathrm{M})$ across conditions. Initially, we report computations in which $P$ and $M$ were assumed to be independent random variables; subsequently, we consider the case in which they are correlated.

All experiments reported in this article involved two discrimination conditions factorially combined with three or four orientations. To simplify the computations, we considered only the two extreme orientations in each experiment (i.e., upright and upside-down), where the predictions of interference-free overlap are the most extreme. Thus, we computed the predicted 
(a)

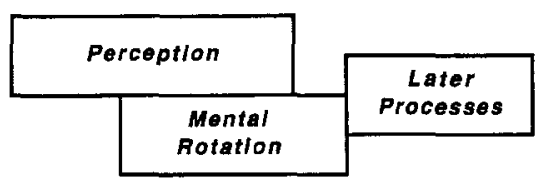

$\operatorname{Time} \underset{T_{0} \quad T_{M}}{+}$

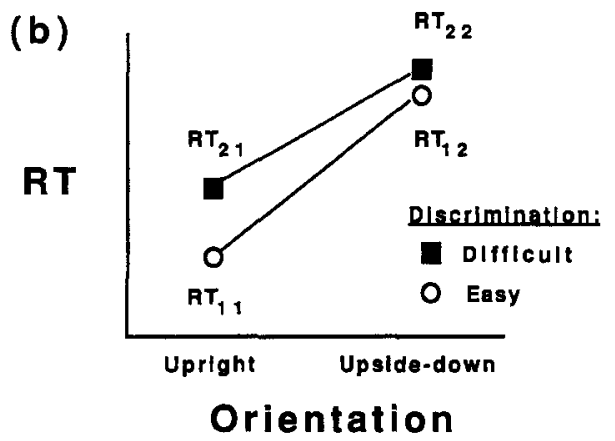

Figure A1. Processing time diagram of an interference-free temporal overlap model and a hypothetical plot of reaction time as a function of discrimination difficulty and orientation.

interaction in a factorial experiment with two orientations and two levels of discriminability (see Figure A1b). We refer to the RTs obtained in discriminability condition $i$ and orientation $j$ as $\mathrm{RT}_{i j}$. Therefore, the model predicts,

$$
\mathrm{RT}_{i j}=\max \left(\mathrm{P}_{i j}, \mathrm{M}_{i j}\right) .
$$

To obtain mean $\mathrm{RT}_{i j}$ s using Equation 2, we first needed to assume a specific shape for the distributions of $\mathrm{P}_{i j}$ and $\mathrm{M}_{i j}$. We chose to use gamma distributions for both, because this distribution provides a reasonable model for a stochastic latency mechanism (Luce, 1986) and it produces roughly the amount of skew observed in the data. (Later in this Appendix, we describe results of related computations using normal rather than gamma distributions.)

\section{Estimation of Model Parameters}

We first determined reasonable limits for the parameters of the model (i.e., the means and variances of each $\mathbf{P}_{i j}$ and $\mathbf{M}_{i j}$ ) from the observed data and then computed predicted underadditivities using a large number of combinations of reasonable parameter values. (Note that we refer to the parameters $\mathrm{M}_{i j}$ and $\mathrm{P}_{i j}$ in terms of means and variances for ease of discussion. Although the parameters typically used to describe gamma distributions do not correspond directly to means and variances, the mean and variance uniquely determine these parameters.) To give the model every chance of predicting underadditivities near the observed value, the limits on parameter values were chosen liberally - that is, we examined parameter combinations in which some or all of the parameters had relatively extreme values. Therefore, when no combination of parameter values predicted an underadditivity of approximately the size we observed (i.e., within sampling error of the observed value), we could be confident that the model was inappropriate for that data set.

We applied several restrictions when choosing parameter values for the gamma distributions. The first restriction was that the mean stage finishing times for upright letters in the easy discrimination condition (i.e., $\mathrm{M}_{11}$ and $\mathrm{P}_{11}$ ) be consistent with data from the single-task control experiments in which subjects performed only the stimulus identification task or the mirror/ normal task (one of these control experiments is described in the Discussion section of Experiment 1). Identification-only control experiments revealed that the time required to make discriminative responses based on shape or color in the present experiments was about $500 \mathrm{msec}$. Assuming that between 200 and $400 \mathrm{msec}$ of this time was due to response processes, then at least $100 \mathrm{msec}$, but at most $300 \mathrm{msec}$, of this time was due to discrimination processes. Across computations, therefore, we let the mean duration of perception in the easy discrimination condition $\left(P_{11}\right)$ vary from 100 to $300 \mathrm{msec}$ in steps of 10 msec. Similarly, control experiments revealed that mirror/ normal responses to upright stimuli require about $850 \mathrm{msec}$. If we subtract between 200 and $550 \mathrm{msec}$ for response processes in this mirror/normal task, then the finishing time of mental rotation must be between 300 and $650 \mathrm{msec}$. Therefore, we let the mean finishing time of mental rotation for upright stimuli in the easy discrimination condition $\left(\mathrm{M}_{11}\right)$ vary from 300 to $650 \mathrm{msec}$ in steps of $10 \mathrm{msec}$.

After estimating the means of M11 and P11, we estimated the corresponding variances by assuming that each variance was equal to the mean times a constant. (It is often observed that the standard deviation [rather than the variance] of RTs varies linearly with the mean [Chocholle, 1945; Myerson, Widaman, \& Hale, 1990]. We therefore conducted an additional set of simulations assuming that the standard deviation is a linear function of the mean. The predicted deviations from additivity were slightly greater under these conditions [i.e., under this assumption, it would be easier to reject interferencefree overlap models], but the differences were fairly small.) Across all of the experiments and associated control experiments, the ratio of variance to mean ranged from 40 to 140 , so we let this parameter vary from 40 to 140 in steps of 10 .

A further restriction was that the finishing time of the discrimination process $\left(\mathrm{P}_{i j}\right)$ be independent of $j$, the orientation of the stimulus letter. And similarly, the finishing time of mental rotation $\left(\mathrm{M}_{i j}\right)$ was restricted to be independent of $i$, the difficulty of the discrimination process, except for certain conditions of Experiments 1-3, as described later. Using this restriction, we were able to immediately determine $M_{21}$ and $P_{12}$ using the estimates of $M_{11}$ and $P_{11}$.

Next, we chose $M_{12}$ and $P_{21}$ so that the means and variances of the resulting $\mathrm{RT}_{i j} \mathrm{~s}$ match the experimental data in terms of the simple main effects of orientation $\left(\mathrm{RT}_{12} \mathrm{vs}\right.$. $\left.\mathrm{RT}_{11}\right)$ and discriminability $\left(\mathrm{RT}_{21}\right.$ vs. $\left.\mathrm{RT}_{11}\right)$. For instance, if an experiment produced a $100-\mathrm{msec}$ discriminability effect for upright letters, then the $\mathrm{M}_{i j}$ and $\mathrm{P}_{i j}$ should also produce a $100-\mathrm{msec}$ discriminability effect. Once these estimates were obtained, we could determine $\mathrm{M}_{22}$ and $\mathrm{P}_{22}$ by applying the restrictions described in the previous paragraph.

One additional parameter was included in the computations for Experiments 1-3. This parameter allowed for the possibility that some aspect of the mirror/normal discrimination proceeds more (or less) rapidly for visually similar letters. We include this parameter because control experiments involving only mirror/normal judgments revealed that the subjects consistently responded faster when the stimuli were visually similar. It might be that subjects begin rotating sooner in these conditions, or perhaps the mirror/normal discrimination proceeds more swiftly. It is important to note, however, that the silar letters; visual similarity simply reduced $\mathrm{RT}$ at all orientations by a constant amount. To account for this effect, we let 
the duration of the mental rotation stage for each orientation condition $\left(\mathrm{M}_{i j}\right)$ vary with the difficulty of the perceptual discrimination. We refer to this parameter as $\mathrm{M}_{\text {offset }}$, where

$$
\mathrm{M}_{\text {offset }}=\mathrm{M}_{1 j}-\mathrm{M}_{2 j}
$$

for all $j \mathrm{~s}$.

In a control experiment using the stimuli of Experiment 1 but requiring only a mirror/normal judgment, response times were $63 \pm 27 \mathrm{msec}$ faster when the perceptual discrimination was difficult than when it was easy. Therefore, in Experiment 1 (and in Experiment 2, which was similar), we let $\mathrm{M}_{\text {offset }}$ vary from 36 to $90 \mathrm{msec}$ in steps of $6 \mathrm{msec}$. Experiment 3, however, employed different stimuli and a different response mapping than did Experiments 1 or 2, and it is not clear whether the $M_{\text {offset }}$ is needed for this experiment (i.e., $M_{\text {offset }}$ might be zero). Consequently, we allowed $M_{\text {offset }}$ for Experiment 3 to take on values between 0 and $90 \mathrm{msec}$ in steps of $6 \mathrm{msec}$. Naturally, we assumed $M_{\text {offset }}$ was zero for Experiments 4-6 involving color discriminations; visual similarity of stimulus color should have no effect on the duration of the mirror/normal discriminations.

\section{The Computation of Mean RTs}

For every combination of parameter values, we determined mean $\mathrm{RT}_{i j} \mathrm{~s}$ according to Equation 2 using numerical methods. From Equation 2 and the assumption that $P$ and $M$ are independent, it follows that

$$
F_{\mathrm{RT}}(t)=F_{\mathrm{P}}(t) * F_{\mathrm{M}}(t)
$$

for all $t \mathrm{~s}$, where the $F$ s are the cumulative probability distributions for the random variables indicated by the subscripts. Given that $\mathbf{P}$ and $\mathrm{M}$ were assumed to be gamma distributions with parameter values determined by the particular combination of means and variances being examined, we could compute $F_{\mathrm{p}}(t)$ and $F_{\mathrm{M}}(t)$ and hence obtain $F_{\mathrm{RT}}(t)$. This was differentiated numerically to obtain the probability density of RT, $f_{\mathrm{RT}}(t)$, which was then numerically integrated to obtain the mean, E[RT].

Having computed E[RT] for each condition, we then computed the predicted interaction, defined as the deviation from additivity $\left(E\left[R_{21}\right]-E\left[R T_{11}\right]\right)-\left(E\left[R T_{22}\right]-E\left[R T_{12}\right]\right)$. These procedures generated 83,160 predicted interactions each for Experiments 1 and 2 (i.e., one for each combination of parameter values), 133,056 for Experiment 3, and 8,316 each for Experiments 4-6. For each experiment, we summarized the computations by finding the minimum and maximum predicted deviations from additivity and then checked to see whether the observed interaction fell within the range of interactions computed from the model. For example, in Experiment 2, the minimum and maximum predicted deviations from additivity were 113 and 162 , respectively, whereas the observed deviation was only $52 \pm 39 \mathrm{msec}$. Because the observed deviation from additivity was less than that predicted by the model with any combination of plausible parameter values, even after allowing for sampling error, we concluded that the interference-free overlap model was not compatible with the small underadditivity observed in this experiment. The results of these computations for Experiments 1-6, along with the observed values and their confidence intervals, are shown in Table 1 . Note that the interference-free overlap model could be rejected in Experiments 2, 3, 5, and 6 but not in Experiments 1 and 4 .

\section{Extensions of the Computations}

Nonindependence of stage times. In the computations described above, we assumed that the durations of mental rotation and perception on each trial were independent, but we also conducted additional analyses in which the durations were allowed to be positively or negatively correlated. These analyses were carried out by simulation rather than computation, because we could not derive the equations for the desired means with correlated random variables.

To generate a strong positive correlation, we randomly chose a value from one probability distribution, computed its cumulative probability, and then found the value from the second distribution with the same cumulative probability. The maximum of these two values was taken as the value of RT for one trial, and the same procedure was repeated for many trials. We calculated the mean and standard deviation of these RT values after each run of 1,000 trials, and the simulation was stopped when the standard error of the running mean RT was $1 \mathrm{msec}$ or less. The final value of the running mean was then taken as the estimate of the E[RT] for that condition. Similarly, to simulate negative correlations, we randomly chose a value from one distribution, computed its cumulative probability, and then found the value from the other distribution with the complementary cumulative probability (i.e., one minus the cumulative probability of the first). These two procedures for generating correlated observations yield the maximum and minimum theoretical correlations for the gamma distribution (Whitt, 1976).

We found that positive correlations tend to increase the predicted deviations from additivity by $10-30 \mathrm{msec}$, whereas negative correlations tend to decrease the predicted deviations by $5-30 \mathrm{msec}$. Because the experiments reported in this paper tend to show smaller deviations from additivity than are predicted by interference-free temporal overlap (see Experiments $2,3,5$, and 6), positive correlations tend to make the interference-free overlap model fit even worse, but negative correlations make it fit better. However, even with the extreme negative correlations considered here, interference-free temporal overlap models still predict much larger deviations from additivity than were observed in several of the experiments.

Normal distributions. We conducted an additional set of computations using normal, rather than gamma, distributions of stage durations. This produced essentially the same deviations from additivity as the gamma distribution.

(Manuscript received July 28, 1994; revision accepted for publication October 17,1994 .) 